\title{
A capacitive differential pressure sensor with polyimide diaphragm
}

\section{Pedersen $\dagger$, M G H Meijerink, W Olthuis and P Bergveld}

\author{
MESA Research Institute, University of Twente, PO Box 217, NL-7500 AE
} Enschede, The Netherlands

Presented on 22 October 1996, accepted for publication on 8 April 1997

\begin{abstract}
A capacitive differential pressure sensor has been developed. The process used for the fabrication of the sensor is IC-compatible, meaning that the device can potentially be monolithically integrated on one chip with a suitable signal conditioning circuit. A sensor for a differential pressure range of \pm 1 bar was fabricated and tested with a frequency modulated detection circuit, and good agreement was found with the theoretical model of the sensor. A nominal sensitivity $\Delta C / C$ of $18 \%$ has been measured for a positive differential pressure of 1 bar.
\end{abstract}

\section{Introduction}

It is well acknowledged that the capacitive detection principle is superior to other techniques concerning sensitivity and power consumption. However, a major problem with this type of sensor is that the source capacitance is very small, causing the sensitivity to parasitics and noise to be relatively high. The best approach to minimize these effects is to make the electrical connections between the sensor and the signal conditioning circuit as short as possible. The most extreme implementation of this is to integrate the sensor on the same chip as the signal conditioning circuit. The problems arising from this desire to integrate sensor and electronics are, however, very complicated. If a sensor is to be made on the same piece of silicon as, for instance, CMOS circuits, one has to consider the compatibility between the sensor process and the CMOS process. It is well known that important parameters, such as threshold voltages and $g_{m}$ factors of the CMOS components are very sensitive to variations or changes in the fabrication process. Therefore, the introduction of any additional process steps for the fabrication of the sensor must be carefully considered in order to maintain the device properties of the CMOS circuits. In this paper we report on a capacitive pressure sensor, which may be fabricated directly on substrates already containing CMOS circuits. The fabrication process of the sensor only contains low-temperature $\left(<300{ }^{\circ} \mathrm{C}\right)$ spin-on, evaporation, and etching processes, which are all considered to be harmless to CMOS components. The fabrication process described was derived from previous developments on silicon condenser microphones [1].

\footnotetext{
$\dagger$ Tel: +31 53 4892722, Fax: +31 53 4892287. E-mail address: m.pedersen@eltn.utwente.nl
}

\section{Sensor structure and simulation}

The structure of the sensor is shown schematically in figure 1. It essentially consists of a polyimide diaphragm on a silicon substrate. Between the diaphragm and substrate, an air gap has been created by deposition and subsequent removal of an aluminium sacrificial layer. A metal electrode is attached to both the diaphragm and the substrate. When, in operation, a pressure difference is applied across the diaphragm, it deflects due to the load and a change of electrical capacitance between the electrodes may be observed.

The theoretical behaviour of the sensor can be separated into two parts. One concerns the mechanical characteristics, which determine the deflection properties of the diaphragm under the load of an external pressure. The second part relates to the translation of the mechanical deflection of the diaphragm into a change of capacitance between the two electrodes. The polyimide diaphragm has a builtin tensile stress of about $40 \mathrm{MPa}$. For such a value of built-in stress, it may be assumed that the deflection of the diaphragm is dominated by this stress, and that additional stress introduced from bending may be neglected. The error introduced by this assumption can be compensated for by a linear matching factor $K$. For deflections much smaller than the thickness of the diaphragm, the deflection profile can be approximated by [2]:

$$
w(x, y)=w_{0} \cos \left(\frac{\pi x}{a}\right) \cos \left(\frac{\pi y}{a}\right)
$$

where $a$ is half the side length of the diaphragm, $x$ and $y$ are principal directions with the origin in the centre of the diaphragm, and $w_{0}$ is the centre deflection given by:

$$
w_{0}=K 0.3284 \frac{p a^{2}}{\sigma h_{d}}
$$

in which $\sigma$ is the built-in stress, $h_{d}$ is the thickness of the diaphragm, $p$ is the applied pressure, and $K$ is the matching 


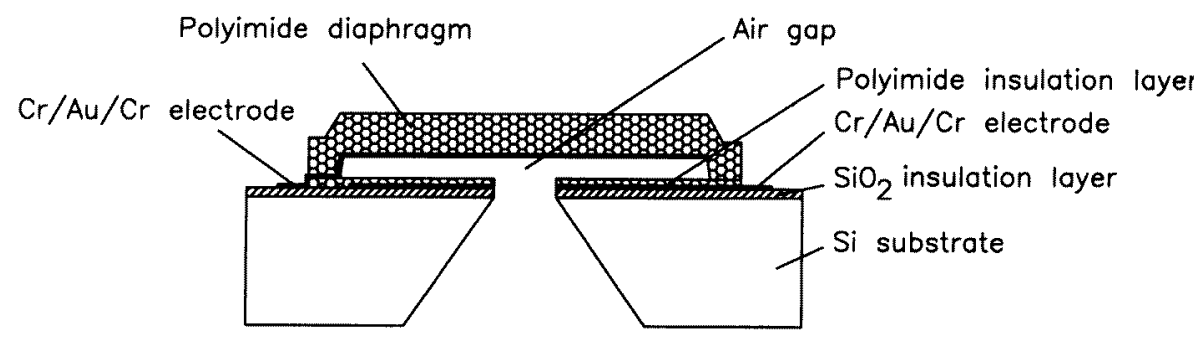

Figure 1. A cross sectional view of the capacitive differential pressure sensor.

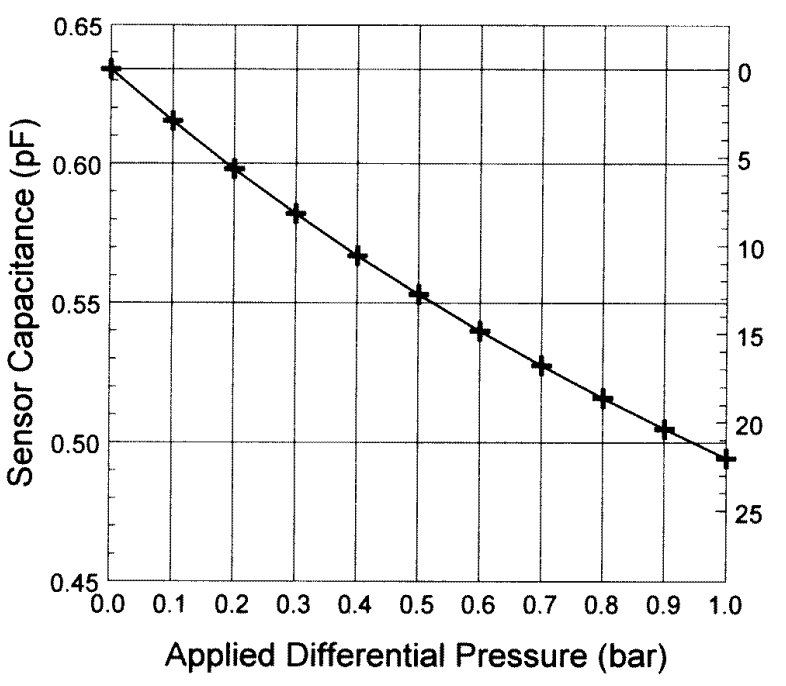

Figure 2. Simulated sensor capacitance versus applied differential pressure.

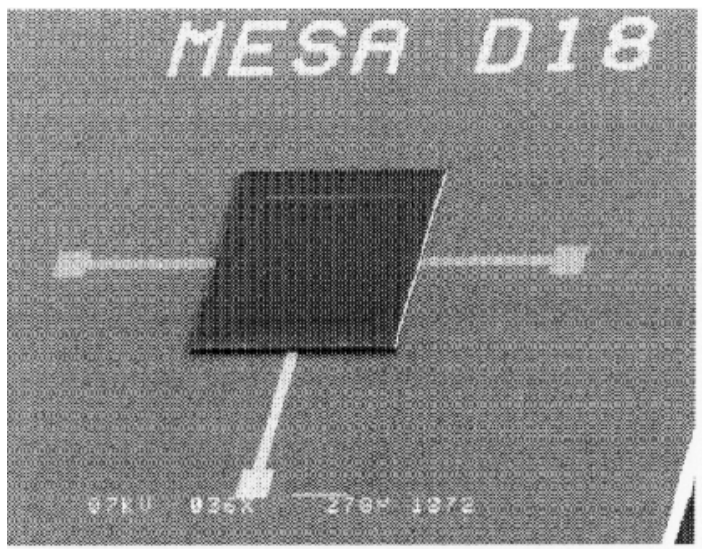

Figure 3. SEM photograph of a completed pressure sensor.

factor. This factor was determined by comparing the simple model with a more complex model [3], which includes both bending and built-in stresses. For a diaphragm of side length $700 \mu \mathrm{m}$ and thickness $16 \mu \mathrm{m}$, a matching factor of 0.57 was calculated.

The capacitance of the sensor can be derived by integration over the surfaces of the two electrodes, of which one is flat (the substrate) and one has the deflection profile of the diaphragm. The capacitance is given by:

$$
C_{\text {sensor }}=\varepsilon_{i} \varepsilon_{0} \int_{-a}^{a} \int_{-a}^{a} \frac{1}{h_{i}+\varepsilon_{i}\left[h_{g}+w(x, y)\right]} \mathrm{d} x \mathrm{~d} y
$$

where $\varepsilon_{i}$ and $h_{i}$ are the relative permittivity and the

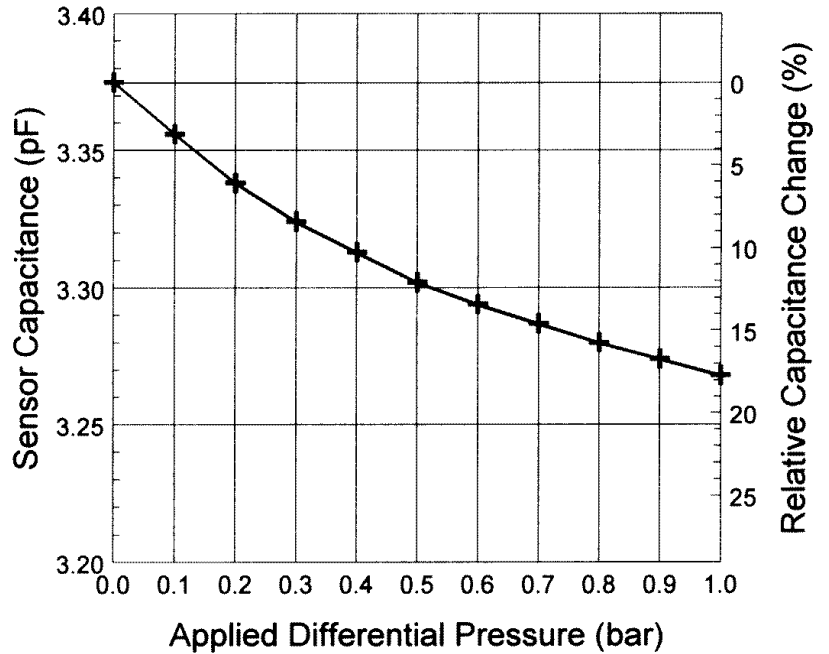

Figure 4. Measured sensor capacitance versus applied differential pressure.

thickness of the polyimide insulation layer respectively, and $h_{g}$ is the height of the air gap.

For the sensor, a diaphragm with a side length of $700 \mu \mathrm{m}$ and a thickness of $16 \mu \mathrm{m}$ was chosen. The thickness of the insulation layer was $0.5 \mu \mathrm{m}$, and the height of the air gap was $5 \mu \mathrm{m}$. According to (2), the diaphragm will have a deflection of $3.6 \mu \mathrm{m}$ for a pressure load of 1 bar. Since this deflection is smaller than the height of the air gap, the sensor can work with both negative and positive pressure differences. Figure 2 shows the a simulation result of the sensor capacitance as a function of the applied differential pressure. As can be seen from figure 2, the theoretical sensor capacitance decreases by more than $20 \%$ in the pressure range from 0 bar to 1 bar.

\section{Measurements and results}

Devices with the dimensions stated above were fabricated using a fabrication process described elsewhere $[1,4]$. A SEM photograph of the front (polyimide diaphragm) of the sensor is shown in figure 3. Since the capacitance of the sensor is very small $(\approx 0.63 \mathrm{pF})$, the influence of the stray capacitance from the interconnection wires is significant. To illustrate this, a measurement on a completed device is shown in figure 4. The sensor was mounted on a test chuck, whereby a pressure could be applied to the backside. The sensor capacitance was measured using a HewlettPackard 4194A Impedance Analyzer. As can be seen from figure 4 , the measured capacitance at $p=0(\approx 3.37 \mathrm{pF})$ 


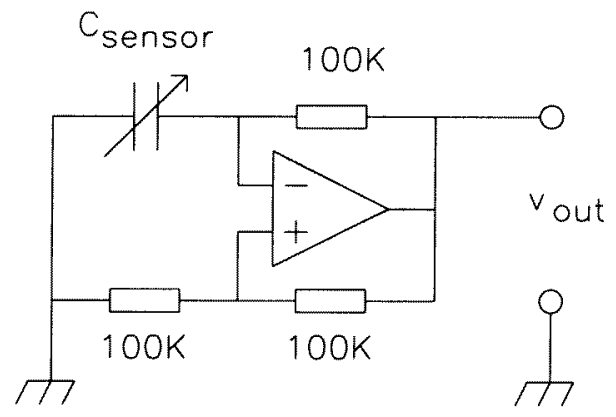

(a)

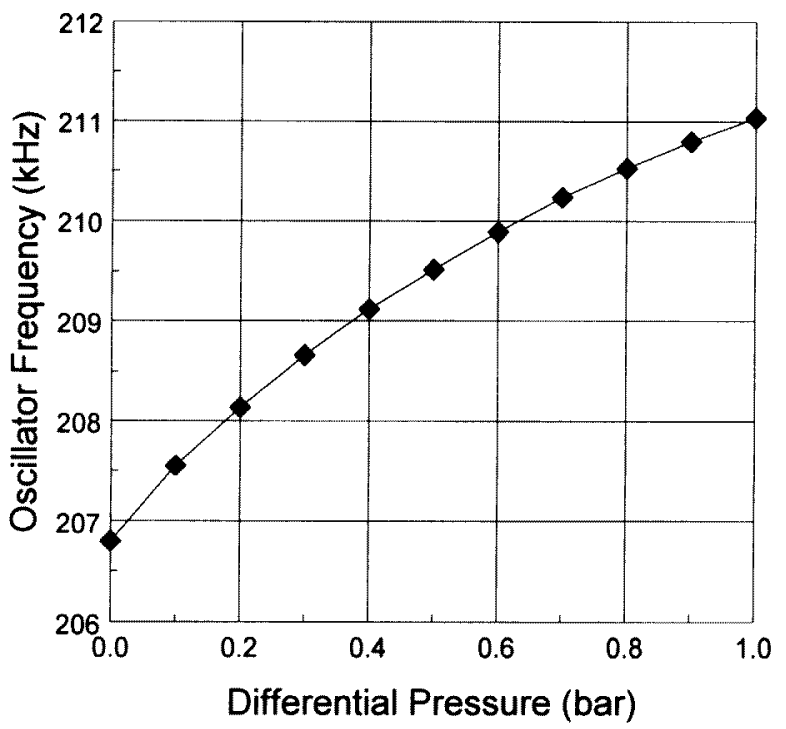

(b)

Figure 5. Oscillator detection circuit (a) and measured output frequency versus applied pressure $(b)$.

is much larger than the theoretical value $(\approx 0.63 \mathrm{pF})$. However, if one considers this extra capacitance $(\approx 2.74 \mathrm{pF})$ to be caused by a parasitic capacitance in parallel with the sensor, the corrected measured relative capacitance change $(\triangle C / C=18 \%)$ is in good agreement with the theory $(\triangle C / C=22 \%)$. The remaining differences between measurements and theory can be explained by deviations of the assumed built-in stress in the polyimide diaphragm. Furthermore, the sensor was tested in a simple oscillator circuit (figure $5(a)$ ) in which the sensor capacitance modulates the frequency of the output of the op-amp. Figure $5(b)$ shows the frequency of the output signal as a function of the applied pressure. As expected, the frequency shows an increase inversely proportional to the decreasing sensor capacitance (figure 4).

\section{Conclusions and discussion}

A capacitive differential pressure sensor containing a polyimide diaphragm has been developed. The fabrication process of the sensor is IC-compatible, and can be made directly on substrates with completed integrated circuits. The sensor was tested and good agreement was found with the theoretical performance. With the introduction of polyimide in the sensor, the stability of the polyimide becomes critical for the sensor performance. It is well known that polyimide, like all polymers, possesses viscoelastic properties in the form of creep and relaxation. However, since the glass transition temperature is high $\left(\approx 400^{\circ} \mathrm{C}\right)$, these effects are expected to be very slow acting. Furthermore, the stress generated in the diaphragm from a pressure load of 1 bar is small $(<0.5 \mathrm{MPa})$. Accordingly, no drift relating to the sensor was detected for a pressure load of 1 bar over a period of one day. Furthermore, pressure measurements were been performed over ten cycles, and no hysteresis was observed. The sensitivity to temperature is also important, and since the coefficient of thermal expansion (CTE) is $\approx 50 \mathrm{ppm} \mathrm{K}-1$ [5] for polyimide, compared to $\approx 3 \mathrm{ppm} \mathrm{K}^{-1}$ for silicon, a reduction of the built-in stress in the diaphragm will occur for increasing temperatures. Assuming that Young's modulus of polyimide is $3 \mathrm{GPa}$ [5], this yields a stress reduction in the diaphragm of approximately $0.25 \mathrm{MPa}{ }^{\circ} \mathrm{C}^{-1}$. Recent developments in polymer science, however, have demonstrated the possibility of synthesizing polyimides with thermal properties matched to silicon [6]. Therefore, in the future it may be possible to realize structures with less sensitivity to temperature.

\section{References}

[1] Pedersen M, Olthuis W and Bergveld P 1997 A silicon condenser microphone with polyimide diaphragm and backplate Sens. Actuators A accepted

[2] Timoshenko S P and Woinowsky-Krieger S 1959 Theory of Plates and Shells 2nd edn (New York: McGraw-Hill)

[3] Pedersen M, Olthuis W and Bergveld P 1995 Modelling and simulation of the quasi static behaviour of silicon condenser microphones Proc. MME'95 (Copenhagen, Denmark) September 1995

[4] Pedersen M, Meijerink M G H, Olthuis W and Bergveld P 1997 An IC-compatible polyimide pressure sensor with capacitive read-out Sens. Actuators A accepted

[5] Jensen R J, Cummings J P and Vora H 1984 Copper/polyimide materials system for high performance packaging IEEE Trans. Compon. Hybrids Manuf. Technol. 7384

[6] Rohde O, Smolka P, Falcigno P A and Pfeifer J 1992 Novel auto-photosensitive polyimides with tailored properties Polym. Eng. Sci. 32 1623-9 